\title{
Varicella pneumonia: A serious complication of varicella in adults
}

\section{Abdellah Dah Cherif, Sara Elloudi, Mohammed Chouche, Younes Barbach, Hanane Baybay, Fatima Zahra Mernissi}

Dermatology Department, University Hospital Hassan II, Fez, Morocco

Corresponding author: Dr. Abdellah Dah Cherif, E-mail: abdellahdahcherif@gmail.com

\begin{abstract}
Pneumonia caused by varicella infection is a serious and severe complication of the disease when it occurs in adults. We report one case of a varicella pneumonia in a 41 year-old non-immunocompromised man. They were admitted to hospital for acute respiratory failure, not requiring intensive care, associated with exanthema. Thoracic imaging revealed bilateral and diffuse alveolar-interstitial opacities in both lung fields. Thrombocytopenia, hepatic cytolysis and an increase in lacticodeshydrogenase were noted. The patients made a full recovery with anti-viral therapy and oxygen.
\end{abstract}

Key words: Pneumonia; Varicella; Lung infection; Antiviral treatment 


\title{
La pneumonie varicelleuse: Une complication grave de la varicelle chez l'adulte
}

\section{Abdellah Dah Cherif, Sara Elloudi, Mohammed Chouche, Younes Barbach, Hanane Baybay, Fatima Zahra Mernissi}

Dermatology Department, University Hospital Hassan II, Fez, Morocco

Corresponding author: Dr. Abdellah Dah Cherif, E-mail: abdellahdahcherif@gmail.com

\begin{abstract}
RÉSUMÉ
La varicelle est une maladie éruptive très fréquente et habituellement bénigne chez l'enfant sain. Chez l'adulte, son évolution peut être émaillée de complications graves telles que la pneumonie varicelleuse. Au cours des dernières décennies, on a noté en Europe et aux États-Unis une augmentation significative de l'incidence de la varicelle chez les adultes et parallèlement, une augmentation du nombre d'hospitalisations et du taux de mortalité. Lincidence de l'atteinte pulmonaire est estimée entre 5 et $50 \%$. on rapporte un cas de pneumonie varicelleuse avec une détresse respiratoire minime chez un adulte immunocompétent.
\end{abstract}

Mots clés: Pneumonie; Varicelle; Infection respiratoire; Antiviral

\section{INTRODUCTION}

La varicelle est une maladie éruptive très fréquente et habituellement bénigne chezl'enfant sain. Chezl'adulte, son évolution peut être émaillée de complications graves telles que la pneumonie varicelleuse [1].

Au cours des dernières décennies, on a noté en Europe et aux États-Unis une augmentation significative de l'incidence de la varicelle chez les adultes et parallèlement, une augmentation du nombre d'hospitalisations et du taux de mortalité. L'incidence de l'atteinte pulmonaire est estimée entre 5 et $50 \%$. on rapporte un cas de pneumonie varicelleuse avec une détresse respiratoire minime chez un adulte immunocompétent [2].

\section{CASE REPORT}

Un patient de 46 ans, Fallah de profession, sans ATCD pathologique notable en particulier sans cause évidente d'immunodépression, était hospitalisé aux urgence pour une éruption cutanée fébrile, avec une toux sèche et une dyspnée.
L'histoire de sa maladie débutait une semaine avant son admission par l'installation de fièvre, d'une asthénie et d'une toux sèche. Le lendemain, apparaissait une éruption cutanée maculopapuleuse et prurigineuse débutant aux deux avant-bras et au dos. Le diagnostic d'une varicelle était porté vue la notion d'un contage avec son neveu et l'extension des lésions à tout le corps avec apparition de lésions vésiculeuses. Deux jours plus tard, le patient se plaignait d'une dyspnée d'aggravation rapide, accompagnée d'une toux sèche. Il consultait les urgences d'ou son hospitalisation.

Lexamen clinique trouvait une température de $39^{\circ} \mathrm{C}$, une pression artérielle à 120/60 mmHg, une polypnée à 26 cycles/min, une légère cyanose des extrémités, des signes discrets de lutte respiratoire, une saturation percutanée en oxygène à $82 \%$ en air ambiant, une fréquence cardiaque à 100 battements par minute sans signe d'insuffisance circulatoire aiguë et à l'auscultation pulmonaire, des râles crépitants diffus aux deux champs pulmonaires. Léruption cutanée était faite de lésions diffuses à tout le revêtement cutané d'âges différents à type de macules, papules,

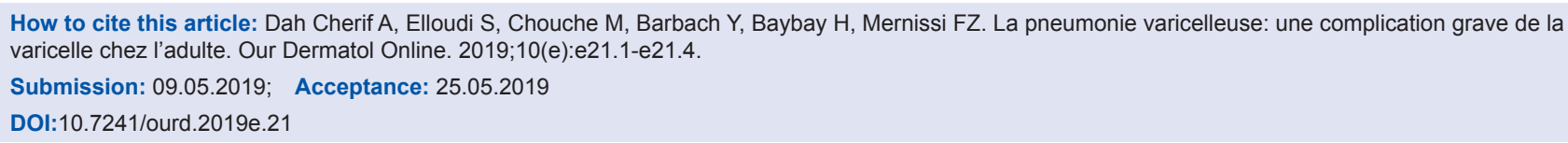


vésicules qui se desséchaient et devenaient croûteuses (Fig. 1). Les données biologiques montraient à la gazométrie artérielle une hypoxémie sévère $(\mathrm{PaO} 2=45 \mathrm{mmHg}, \mathrm{pH}=7,49, \mathrm{SaO} 2=84 \%)$, une hyperleucocytose à 10600 par millimètre cube, une thrombopénie à 100000 par millimètre cube, une CRP à $123 \mathrm{mg} / \mathrm{l}$, une cytolyse hépatique modérée (GOT $4{ }^{*} \mathrm{~N}$, GPT $\left.3.5^{*} \mathrm{~N}\right)$, une hyponatrémie à $121 \mathrm{mEq} / \mathrm{l}$. La sérologie VIH était négative.La radiographie thoracique montrait la présence d'opacités alvéolointerstitielles bilatérales et diffuses aux deux champs pulmonaires (Fig. 2).

Le diagnostic d'une pneumopathie varicelleuse hypoxémiante était porté. Le patient était mis sous traitement antiviral par acyclovir $(10 \mathrm{mg} / \mathrm{kg}$ tous les huit heures) en intraveineux associé à une oxygénothérapie nasale à raison de $5 \mathrm{l} / \mathrm{min}$. Lévolution était favorable avec l'obtention d'une apyrexie après 48 heures de traitement, amélioration de la dyspnée avec correction de l'hypoxémie $(\mathrm{PaO} 2=74 \mathrm{mmHg})$.
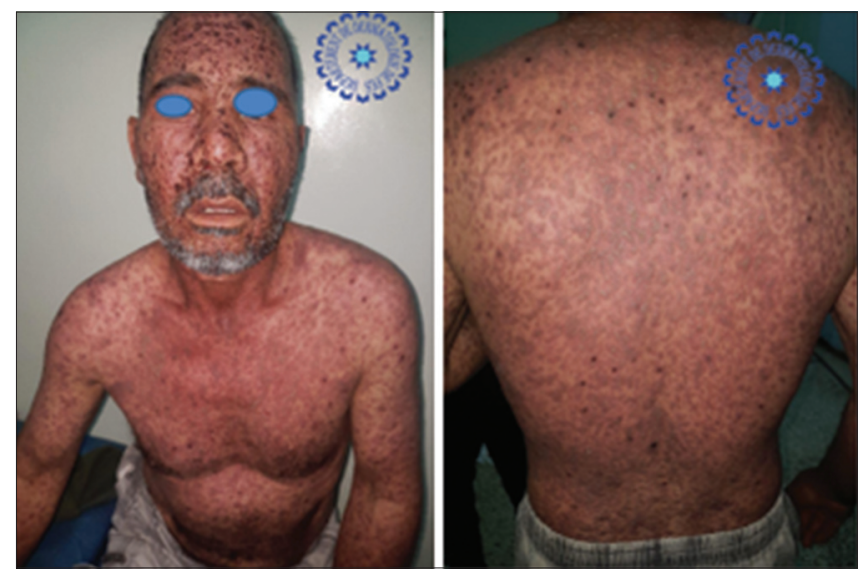

Figure 1: Diffuse maculopapular and vesicular eruption.

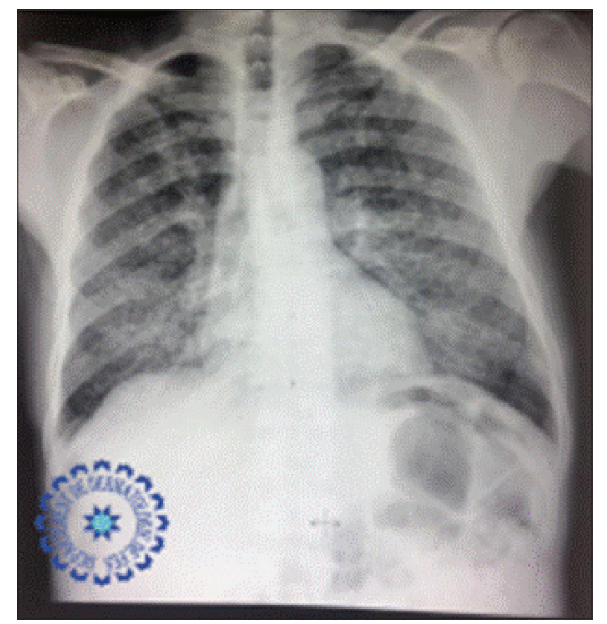

Figure 2: Bilateral and diffuse interstitial alveolar opacities at the two lung fields.

\section{DISCUSSION}

Nous rapportons un cas de patient atteint de pneumonie varicelleuse qui se présenté dans un tableau d'insuffisance respiratoire aiguë minime avec un syndrome alvéolointerstitiel à l'imagerie thoracique. Lévolution était favorable sous traitement antiviral et une oxygénothérapie à fort débit. La pneumonie varicelleuse est la plus fréquente des complications graves de la varicelle chez l'adulte, son incidence est estimée de 16 à $33 \%$ avec une mortalité pouvant atteindre $20 \%$ [1]. D'autres complications dues à la dissémination virale ont été décrites, notamment l'encéphalite, l'hépatite et les troubles de la coagulation [3]. Notre patient a présenté une cytolyse hépatique modérée et une thrombopénie qui ont régressé sous traitement antiviral. Plusieurs facteurs ont été associés au développement de l'atteinte pulmonaire dans la varicelle. Le tabagisme, par ses effets nocifs sur la clairance mucociliaire bronchique et l'altération de l'activité des macrophages alvéolaires, fait augmenter le risque de développer une pneumonie. Limportance de l'atteinte cutanée (> 100 éléments), l'histoire d'un contact étroit avec un sujet infecté, la grossesse et l'immunodépression constituent des facteurs associés avec une incidence élevée de pneumonie [4]. Notre patient présente comme facteurs de risque un tabagisme chronique, un contact étroit avec un sujet infecté et une atteinte cutanée étendue. Les symptômes respiratoires de la pneumonie varicelleuse débutent en général un à sept jours après l'installation de l'exanthème. Elle se manifeste par une toux sèche parfois accompagnée d'une hémoptysie, des douleurs thoraciques, une dyspnée, une fièvre, voire même un tableau de détresse respiratoire aiguë, comme c'était le cas pour notre patient [1]. Le diagnostic de pneumopathie varicelleuse peut être porté sur l'association d'une atteinte pulmonaire, dont les caractéristiques cliniques et radiologiques sont concordantes et d'une éruption cutanée évocatrice de varicelle en l'absence d'une autre cause possible. Limagerie thoracique montre habituellement des nodules de 5 à $10 \mathrm{~mm}$ de diamètre diffus éventuellement confluents et touchant les deux champs pulmonaires; la présence d'adénopathies hilaires et d'épanchement pleural liquidien est non usuelle. Le scanner thoracique en haute résolution confirme la présence de ces nodules pulmonaires plus ou moins bien limités, coalescents associés à des images en verre dépoli, en halo autour de ces nodules ou disséminées en mosaïque. Les images radiologiques se résolvent habituellement une semaine après la disparition des lésions cutanées, mais elles peuvent persister plusieurs mois [5]. Chez notre patient, le diagnostic a été retenu 
devant les symptômes respiratoires survenus 5 jours après l'éruption cutanée très évocatrice de varicelle, les données de l'imagerie thoracique, et l'évolution clinique favorable sous antiviraux. L'endoscopie bronchique chez l'adulte peut mettre en évidence la présence de lésions vésiculeuses de la muqueuse bronchique. Par ailleurs, son incidence et sa signification clinique et pronostique ne sont pas encore bien connues. Dans notre observation, la fibroscopie bronchique n'a pas été faite, car l'évolution était favorable. Le traitement antiviral par acyclovir a été utilisé avec succès pour la première fois dans le traitement de la pneumonie varicelleuse en 1980 [6]. La conférence de consensus en thérapeutique antiinfectieuse a recommandé l'utilisation d'acyclovir par voie intraveineuse à raison de $10 \mathrm{mg} / \mathrm{kg}$ tous les huit heures dans la varicelle compliquée du sujet adulte, en particulier la pneumopathie varicelleuse pendant une durée de sept à dix jours [7]. Peu de séries ont rapporté la mortalité due à la pneumonie varicelleuse. Sur 272 patients atteints, il existait une différence significative relative au taux de mortalité entre les patients traités par acyclovir et les patients non traités (6,1 \% vs 19,1\%). Lutilisation des corticoïdes dans les formes sévères n'a pas fait l'objet d'études d'un niveau de preuves suffisant. Une étude a rapporté leur utilisation dans les formes graves de pneumonie varicelleuse avec un raccourcissement de la durée de l'hospitalisation [6].

\section{CONCLUSION}

- La pneumonie varicelleuse est une complication grave de la varicelle chez l'adulte.
- Notre observation illustre l'importance de l'analyse sémiologique dans l'orientation diagnostique évitant toute exploration abusive.

- Le traitement précoce par l'aciclovir améliore le pronostic.

\section{REFERENCES}

1. Senneville E, Infections. À VZV: formes de l'adulte sain et de l’immunodéprimé. Med Mal Infect. 1998;28:791-9.

2. Mohsen AH, Mckendrick M. Varicella pneumonia in adults. Eur Respir J. 2003;21:886-91.

3. Mohsen AH, Peck RJ, Mason Z, Mattock L, Mckendrick M. Lung function tests and risk factors for pneumonia in adults with chickenpox. Thorax. 2001;56:796-9.

4. Richaud C, Ngo MT, Agbessi CA, Boru B, Elkharrat D, Chinet T. Atteinte bronchique au cours d'une pneumonie varicelleuse chez un adulte immunocompétent. Rev Mal Respir. 2008;25:59-62.

5. Senneville E, Rivaud E, Valette M, Maulin L. Paramètres non pulmonaires associés à la pneumonie varicelleuse de l'adulte sain. Med Mal Infect. 2000;30:211-6.

6. CEDEF et al. Infections à herpès virus de l'enfant et de l'adulte immunocompétents: varicelle et zona. Ann Dermatol Venereol. 2008;135:F25-F31.

7. 11 éme conférence de consensus en thérapeutique anti-infectieuse de la SPILF: prise en charge des infections à VZV. Med Mal Infect.1998;28:1-8.

8. El-Daher N, Magnussen CR. Varicella pneumonitis: clinical presentation and experience with acyclovir treatment in immunocompetent adults. Int J Infect Dis. 1998;2:147-51.

9. Van der Meer JW, Thompson J, Tan WD, Versteeg J. Treatment of chickenpox pneumonia with acyclovir. Lancet 1980;2:473-4.

Copyright by Abdellah Dah Cherif, et al. This is an open access article distributed under the terms of the Creative Commons Attribution License, which permits unrestricted use, distribution, and reproduction in any medium, provided the original author and source are credited.

Source of Support: Nil, Conflict of Interest: None declared. 